\title{
Reliable and Efficient Algorithm for Broadcasting in Asymmetric Mobile Ad hoc Networks
}

\author{
M.Murugesan \\ Associate Professor/IT \\ K.S.R.College of Engineering \\ Tiruchengode, Namakkal-637 215
}

\author{
A. Krishnan \\ Dean \\ K.S.Rangasamy College of technology, \\ Tiruchengode, Namakkal-637 215
}

\begin{abstract}
Network wide broadcasting is a fundamental operation in ad hoc networks. In broadcasting, a source node sends a message to all the other nodes in the network. Unlike in a wired network, a packet transmitted by a node in ad hoc wireless network can reach all neighbors. Therefore, the total number of transmissions (Forwarding nodes) used as the cost criterion for broadcasting. The broadcast operation, as a fundamental service in mobile ad hoc networks (MANETs), is prone to the broadcast storm problem if forwarding nodes are not carefully designated. The forward node selection has been studied extensively in undirected graphs in which each node has the same transmission range. In practice, the transmission ranges of all nodes are not necessarily equal. This thesis proposes a reliable and efficient localized broadcasting algorithm using 2-hop neighborhood information more effectively to reduce redundant transmissions in asymmetric Mobile Ad hoc networks that guarantees full delivery. Among the 1-hop neighbors of the sender, only selected forwarding nodes retransmit the broadcast message. Forwarding nodes are selected such a way that to cover the uncovered 2-hop neighbors. The retransmissions of the forwarding nodes are received by the sender as the confirmation of their reception of the packet. The non forwarding 1-hop neighbors of the sender only acknowledge the reception of the broadcast. If the sender does not detect all its forwarding nodes retransmissions and non forwarding nodes acknowledgements, it will resend the packet until the maximum number of retries is reached. Simulation results show that the proposed broadcast algorithm provides good broadcast delivery ratio with low overhead and minimum latency.
\end{abstract}

\section{General Terms}

Wireless Mobile Ad hoc networks, broadcasting.

\section{Keywords}

Broadcasting, forwarding node set, mobile ad hoc networks, efficient.

\section{INTRODUCTION}

In areas where there is little or no communication infrastructure or the existing infrastructure is inconvenient to use, wireless mobile users may still be able to communicate through the formation of an ad hoc wireless network. An ad hoc wireless network is a collection of wireless mobile hosts forming a temporary network without the aid of any centralized administration or standard support services. In such a network, each mobile node operates not only as a host but also as a router.
The applications of ad hoc wireless networks range from military use in battlefields, personnel coordinate tools in emergency disaster relief, to interactive conferences that temporarily formed using PDAs. The broadcast operation is the most fundamental role in ad hoc networks because of the broadcasting nature of radio transmission: When a sender transmits a packet, all nodes within the sender's transmission range will be affected by this transmission. The advantage is that one packet can be received by all neighbors; the disadvantage is that it interferes with the sending and receiving of other transmissions, creating exposed terminal problem, that is, an outgoing transmission collides with an incoming transmission, and hidden terminal problem, that is, two incoming transmissions collide with each other. In general, broadcasting refers to a process of transmitting a packet so that each node in a network receives a copy of this packet. A straightforward approach for broadcasting is blind Flooding where every node in the network forwards the packet exactly once. Flooding ensures the full coverage of all the network, that is, the broadcast packet is guaranteed to be sent to every node in the network, providing the network is static and connected and the MAC layer of the communication channel is error-free during the broadcast process. However, flooding generates many redundant transmissions. Figure 1 shows a sample network with three nodes.

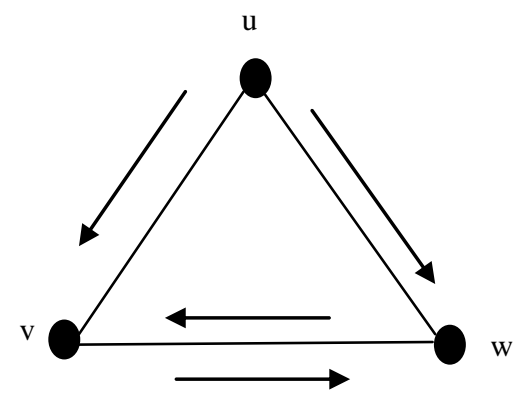

Fig 1. Redundant transmissions by blind flooding

When node $\mathrm{u}$ broadcasts a packet, both nodes $\mathrm{u}$ and $\mathrm{w}$ will receive the packet. Then, $\mathrm{v}$ and $\mathrm{w}$ will rebroadcast the packet to each other. Apparently, there is much broadcast redundancy for blind 'flooding in this case. Transmitting the broadcast packet only by node $\mathrm{u}$ is enough for a broadcast operation. When the size of the network increases and the network becomes dense, more transmission redundancy will be introduced and these transmissions are likely to trigger considerable transmission collision and contention. This is a serious broadcast storm problem that finally falls down the whole network. Since 
MANETs suffer from transmission contention and congestion that are results of the broadcasting nature of radio transmission, it is a major challenge to provide a reliable broadcasting under such dynamic MANETs. This paper aims to reduce broadcast redundancy by decreasing the number of the forward nodes yet still provide high delivery ratio for each broadcast packet in a dynamic environment. A subset of nodes is used to forward the broadcast message and the remaining nodes are still covered (i.e., they are adjacent to forward nodes).ask that authors follow some simple guidelines. In essence, we ask you to make your paper look exactly like this document. The easiest way to do this is simply to down-load a template from [2], and replace the content with your own material.

\section{RELATED WORK}

S. Ni, Y. Tseng, Y. Chen, and J. Sheu [1] discussed the broadcast storm problem. They also analyzed broadcast redundancy, contention and collision in blind flooding. W.Peng and X.Lu [2] proposed Scalable broadcast algorithm in which a node does not rebroadcast the broadcast packet if all of its neighbors have received the packet from previous transmissions. J.P.Sheu,P.K.Hung, and C.S.Hsu[3] provided centralized and distributed aglorithm for broadcasting and experimentally study of their algorithms with respect to collision-free delivery, number of transmissions and broadcast latency. While their centralized algorithm is guaranteed to be collision free,distributed algorithm is not . They do not provide any guarantees with respect to the number of transmissions and latency of the broadcast schedule. A. Qayyum, L. Viennot, and A. Laouiti [4] proposed multipoint relays in which each forward node determine the status of it neighbors based on its partial 2-hop information through node coverage.MPR is source depend, that is the forward node set it dependent on the source of the broadcast . The resultant forward node set depends on many factors, such as the location of neighbors, node priority, message propagation delay and back-off delay .J.Wu and F.Dai [5] proposed a generic distributed broadcast scheme in which a CDS is constructed for a particular broadcast and is dependent on the location of the source.S. Alagar, S. Venkatesan [6] proposed a reliable broadcast (RB) protocol based on flooding. The protocol works as follows:The source broadcasts the message to its 1-hop neighbors. When a node receives the message, it sends an ACK back tothe sender. If the message is a new one, the node retransmits the message; otherwise, it drops the message. If the sender does not receive an ACK from any of its neighbors for a predefined period, it resends the message. J.J. Garcia-Luna-Aceves and Y.X. Zhang[7] use a flooding-based approach that allows the nodes that received the broadcast packet to forward the packet without further notice from the sender. When the source sends the message, it waits for the ACKs from all its neighbors. E. Pagani and G.P. Rossi [8] propose to set up a forwarding tree, which is rooted from the cluster head of the source to each cluster head, based on virtual cluster architecture for a reliable broadcast in MANETs. The broadcast packet is forwarded down the tree from the root source to the leaf nodes and the ACKs are collected by each cluster head and sent up the tree from the leaves to the root. The source retransmits the packet if an error occurs. The algorithm changes to flooding when the rate of topology change of the network becomes high. Lim and Kim[9] provided a dominant pruning
algorithm(DP). Compared to the MPR, the DP excludes the coverage of the forwarded node from the current node's 2-hop neighbor set. Supposing $\mathrm{u}$ is the last forwarded node and $\mathrm{v}$ is a designated forwarding node of $\mathrm{u}, \mathrm{v}$ selects its forwarding node set from $\mathrm{X}=\mathrm{H}(\mathrm{v})-\mathrm{N}(\mathrm{u})$ to covered only by nodes in $\mathrm{N}(\mathrm{u})$ but not by nodes in $\mathrm{H}(\mathrm{v})-\mathrm{N}(\mathrm{u})$. Lou and $\mathrm{Wu}[10]$ proposed a partial dominant pruning algorithm(PDP) to extend the DP by further reducing the number of 2-hop neighbors to be covered by 1-hop neighbors. Peng and Lu [11]proposed a CDS based broadcast algorithm(CDSB). When a node receives a broadcast packet and determines its forwarding nodes with lower node IDs to determine its own forwarding node set. Wei Lou et al., [12] have proposed a simple broadcast algorithm, called Double-Covered Broadcast which takes advantages of broadcast redundancy to improve the delivery ratio in an environment that has rather high transmission error rate.

\section{THE PROPOSED BROADCASTING SCHEME}

\subsection{Network Model}

An Ad hoc network can be modeled using a Directed Graph $\mathrm{G}=(\mathrm{V}, \mathrm{E})$. The node in $\mathrm{V}$ are located in a Euclidean Plane, and each node $\mathbb{D}_{1} € \mathrm{~V}$ has transmission range $\gamma_{i} €\left[\gamma_{\min }, \gamma_{\text {max }}\right]$, where $V_{\text {mim }}$ is the minimum transmission range and $\gamma_{\text {mas }}$ is the maximum transmission range of a network. A directed edge $\left(\mathbb{E}_{\mathfrak{q}}, \mathbb{E}_{\mathfrak{j}}\right) \in \mathbb{E}$ if $\mathrm{d}\left(\mathbb{E}_{\mathfrak{i}}, \mathbb{E}_{\mathfrak{j}}\right) \leq \mathbb{Y}_{i}$ where $\mathrm{d}\left(\mathbb{E}_{\mathfrak{i}}, \mathbb{E}_{\mathfrak{j}}\right)$ denote the Euclidean distance between $\mathbb{E}_{\mathfrak{i}}$ between $\mathbb{E}_{j}$. Such a graphs are called Directed Graphs (DG). An edge $\left(\mathbb{E}_{\mathfrak{q}}, \mathbb{D}_{\mathfrak{j}}\right)$ is bidirectional if both $\left(\mathbb{E}_{\mathbb{q}}, \mathbb{E}_{\mathbb{1}}\right)$ and $\left(\mathbb{E}_{\mathbb{1}}, \mathbb{E}_{\mathbb{q}}\right)$ are in $\mathrm{E}$.

Consider the Fig.2. Due to the different transmission ranges of nodes $\mathrm{u}, \mathrm{v}, \mathrm{w}$, if there is an asymmetric link (u, v) from node $\mathrm{u}$ to $\mathrm{v}$ and symmetric links between $\mathrm{v}$ and $\mathrm{w}$ and between $\mathrm{w}$ and $\mathrm{u}, \mathrm{v}$ realizes the asymmetric link $(\mathrm{u}, \mathrm{v})$ if $\mathrm{v}$ receives the HELLO message from $u$ with $u$ 's 1-hop neighbor set $N(u)=\{w\}$ and finds itself not in $\mathrm{N}(\mathrm{u})$. $\mathrm{v}$ starts a local broadcast REQ to find $u$. Intermediate node $w$ attaches ID and forwards the REQ. When $\mathrm{u}$ receives the REQ, it recognized the asymmetric link $(\mathrm{u}, \mathrm{v})$ and builds the feedback path $(\mathrm{v}, \mathrm{w}, \mathrm{u})$ and informs $\mathrm{v}$ of the feedback path.



Fig.2 A sample network with asymmetric links 


\subsection{An Efficient Forward Node List Selection 5 Algorithm (EFNLA)}

Let $\mathrm{S}$ be the node that determine its forwarding node list FNL(S). N(S) represents the 1-hop neighbor list of S(including $S) . N_{2}(S)$ represents the 2-hop neighbor list of $S($ i.e, the set of nodes that are within two hops from $\mathrm{S}$ ).

$$
\begin{aligned}
& \text { Clearly }\{S\} \subseteq N(v) \subseteq N_{2}(S) \\
& \text { If } v € N(S) \text { then } N(v) \subseteq N_{2}(S)
\end{aligned}
$$

The node (S) of the broadcast operation uses the following algorithm to determine its forwarding node set.

1. Node $S$ computes $X=N(S)-S$ and $\left.U C L(S)=N_{2}(S)\right)-N(S)$ and $\mathrm{FNL}(\mathrm{S})=\varphi$

2. First select those 1-hop neighbor nodes in $\mathrm{X}$ as forward nodes which are the only neighbor of some node in UCL(S). Add these 1-hop neighbor nodes to the FNL(S) and remove from X. Also remove the 2-hop neighbors which are covered by the above 1hop neighbors from UCL(S).

3. Find $w($ in $\mathrm{X}$ ) with maximum effective neighbor degree using $\operatorname{deg}(\mathrm{w})$ which consists of nodes that are in both $\mathrm{N}(\mathrm{w})$ and $\mathrm{UCL}(\mathrm{S})$.

4. $\quad F N L(S)=F N L(S) \cup\{w\}, U C L(S)=U C L(S)-N(w)$ and $X=X-\{w\} \backslash$

5. Repeat step 3 and 4 until UCL(S) becomes empty.

\subsection{Reliable And Efficient Broadcast Algorithm (REBA)}

A reliable broadcast operation requires the packet to be received by all the nodes in the network. But the interference of the transmission of neighbors and the movement of the nodes may cause the failure of some nodes to receive the packet. Therefore, the sender needs to retransmit the packet to increase the delivery ratio of the transmission. follows:

The proposed reliable broadcast algorithm works as

1. When a source broadcast a packet, it selects some neighbors from its 1-hop neighbor set that form its forwarding node list to cover its 2-hop node set.

2. After the forward nodes receive the new broadcast packet, each forward node records the packet, computes its forward nodes and rebroadcasts the packet.

3. The retransmissions of the forwarding nodes are overheard by the sender as the acknowledgement of the reception of the packet. The non forwarding 1-hop neighbors of the sender only explicitly acknowledge the receipt of the packet.

4. The sender waits for a predefined duration to overhear the rebroadcast from its forwarding nodes and to receive ACKs from its non forwarding nodes. If the sender fails to detect all its forwarding nodes retransmission and does not receive all non forwarding nodes ACKs during this duration, it assumes that a transmission failure has occurred for this broadcast and the packet
5. If the sender fails to receive ACKs from all its non forwarding nodes and fails to detect all forwarding nodes retransmission for a threshold number of times, the sender assumes the nodes that do not reply are out of its transmission range and stops further attempts.

6. 6. If the node that misses the packet is a non-forward node, then the missed packet does not cause other missing propagations in the network. On the other hand, if it is a forward node that misses the broadcast packet, this miss may propagate through the network since the neighbors of this forward node will also miss the packet. So when a sender fails to detect retransmission of a forward node after maximum number of retries, it reselects alternative forward node to cover the set which is supposed to be covered by it.

\section{RESULTS AND DISCUSSIONS}

In this section simulation results are presented which demonstrate the performance the proposed algorithm and evaluation of the performance of the algorithm based on Broadcast forwarding ratio and delivery ratio:

Broadcast forwarding ratio is the fraction of the total number of nodes in the network that at least retransmit broadcast packets once for one broadcast operation. Broadcast delivery ratio is the ratio of the number of the nodes that received packets to the number of the nodes in the network for one broadcast operation.

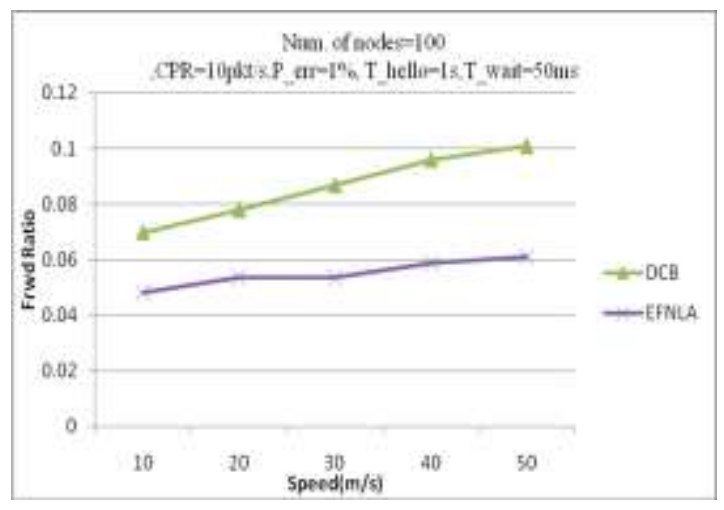

Fig 3. Sensitivity to mobility of the node: Forwarding

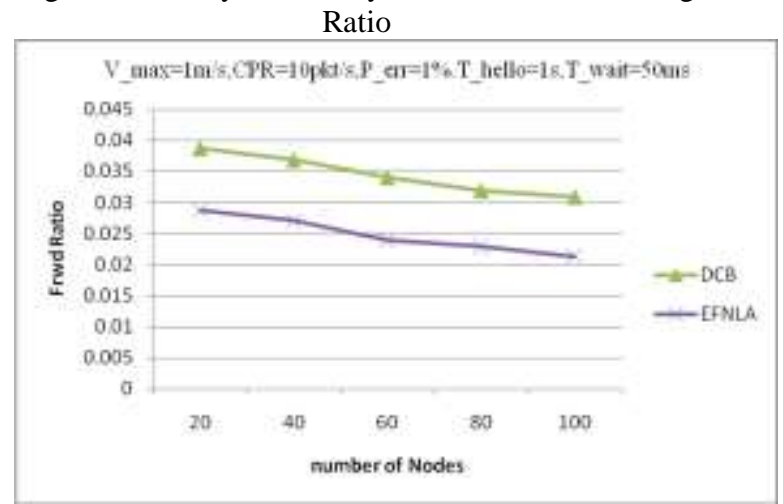

Fig 3. Sensitivity to number of nodes: Delvery Ratio 


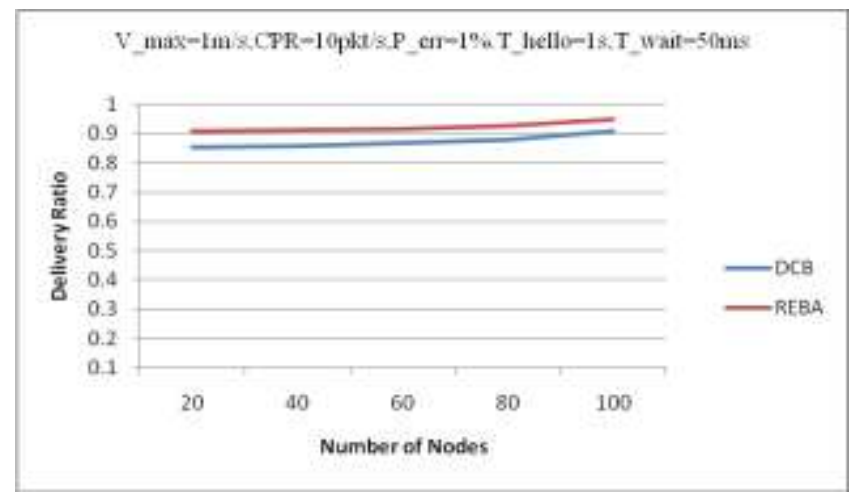

Fig 4. Sensitivity to number of nodes: Deliery Ratio

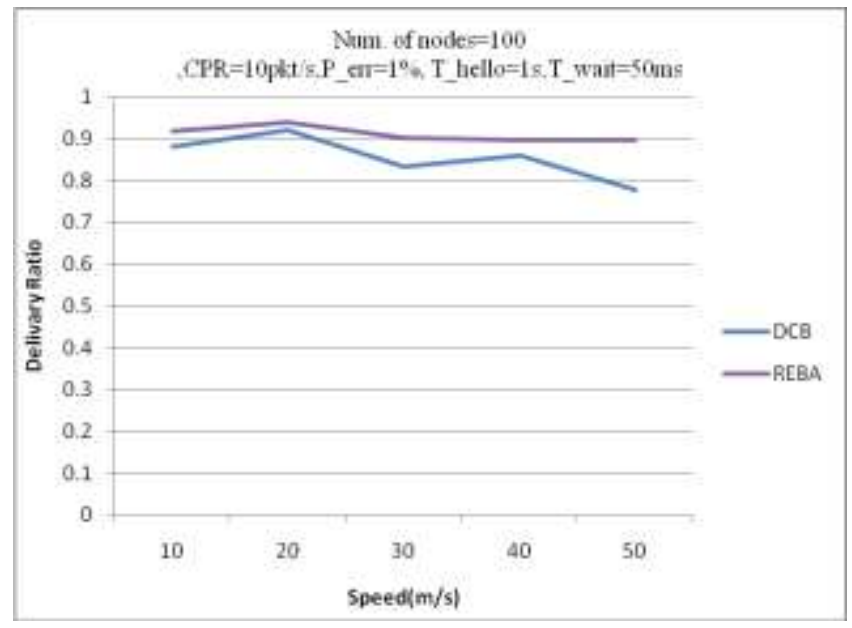

Fig 5. Sensitivity to mobility of the node: Forwarding Ratio

\section{CONCLUSION}

In this paper, an efficient and reliable broadcasting algorithm in Asymmetric Mobile Ad hoc Networks has been proposed which provides high delivery ratio while suppressing broadcast redundancy. This is achieved by only requiring some selected forwarding nodes among the sender's 1-hop neighbor set to forward the packet. The directed DGs can be used to model wireless ad hoc networks, where nodes have different transmission ranges. The simulation results show that the proposed algorithm has high delivery ratio, low forwarding ratio.

\section{REFERENCES}

[1]. S. Ni, Y. Tseng, Y. Chen, and J. Sheu, "The Broadcast Storm Problem in a Mobile Ad Hoc Network," Proc. ACM/IEEE MOBICOM '99, pp. 151-162, Aug. 1999.

[2].W.Peng and X.Lu,"O $\mathrm{n}$ the Reduction of Broadcast Redundancy in Mobile Ad Hoc Networks," Proc.MobiHoc C onf.,pp.129-130,2000.
[3].J.P.Sheu,P.K.Hung, and C.S.Hsu,"Scheduling of broadcasts in multihop wireless networks",ih The Handbook of Ad Hoc Wireless Networks. Boca Raton,FL:CRC Press,2003,pp 483-495

[4].A. Qayyum, L. Viennot, and A. Laouiti, "Multipoint Relaying for Flooding Broadcast Message in Mobile Wireless Networks," Proc. 35th Hawaii Int'l Conf. System Sciences (HICSS-35), pp. 3898-3907, Jan. 2002 .

[5].J.Wu and F.Dai. "A generic distributed broadcast scheme in ad hoc wireless networks".Proc.ofICDCS 2003, pages 460468,2003 .

[6].S. Alagar, S. Venkatesan, and J. Cleveland, "Reliable Broadcast in Mobile Wireless Networks," Proc. Military Comm. Conf. (MILCOM'95), pp. 236-240, Nov. 1995.

7]. J.J. Garcia-Luna-Aceves and Y.X. Zhang, "Reliable Brodcasting in Dynamic Network," Proc. 1996 IEEE Int'l Conf. Comm. (ICC '96),vol. 3, pp. 1630-1634, June 1996.

[8].E. Pagani and G.P. Rossi, "Providing Reliable and Fault Tolerant Broadcast Delivery in Mobile Ad Hoc Networks," Mobile Networks and Applications, vol. 4, pp. 175-192, 1999.

[9] H. Lim and C. Kim, "Flooding in Wireless Ad Hoc Networks," Computer Comm. J., vol. 24, nos. 3-4, pp. 353-363, 2001.

[10] W. Lou and J. Wu, "On Reducing Broadcast Redundancy in Ad Hoc Wireless Networks," IEEE Trans. Mobile Computing, vol. 1, no. 2, pp. 111-123, Apr.-June 2002.

[11] W. Peng and X. Lu, "Efficient Broadcast in Mobile Ad Hoc Networks Using Connected Dominating Sets," J. Software, vol. 12, no. 4, pp. 529-536, 2001.

[12].Wei Lou, Jie Wu, "Towards Broadcast Reliability in Mobile Adhoc Networks with Double Coverage," IEEE Transactions on Mobile Computing, vol. 6, no.2, February 2007.

\section{AUTHORS PROFILE}

Mr. M. Murugesan received his M.E., degree from K. S. Rangasamy College of technology, Tiruchengode. He is now working as a Associate Professor in IT department at K. S. R college of Engineering Tiruchengode. He doing his research in the area of Mobile Adhoc Networks. His research interests includes Mobile Networks, Routing etc.

Dr. A. Krishnan received his $\mathrm{Ph} . \mathrm{D}$ degree in Electrical Engineering from IIT, Kanpur. He is now working as Dean at K.S.Rangasamy College of Technology, Tiruchengode and research guide at Anna University Chennai. His research interests includes Control system, Digital Filters, Power Electronics, Digital Signal processing, Communication Networks etc. He has published more than 250 technical papers at various National/ International Conferences and journals. 